\title{
ESCRT-III mediated cell division in Sulfolobus acidocaldarius - a reconstitution perspective
}

\author{
Tobias Härtel and Petra Schwille* \\ Department of Cellular and Molecular Biophysics, Max Planck Institute of Biochemistry, Martinsried, Germany
}

\author{
Edited by: \\ Marc Bramkamp, \\ Ludwig-Maximilians-University \\ Munich, Germany \\ Reviewed by: \\ Sonja-Verena Albers, Max Planck \\ Institute for Terrestrial Microbiology, \\ Germany \\ Piotr Szwedziak, Medical Research \\ Council Laboratory of Molecular \\ Biology, UK

\section{*Correspondence:} \\ Petra Schwille, Department of Cellular \\ and Molecular Biophysics, Max Planck \\ Institute of Biochemistry, Am \\ Klopferspitz 18, 82152 Martinsried \\ Germany \\ e-mail: schwille@biochem.mpg.de
}

In the framework of synthetic biology, it has become an intriguing question what would be the minimal representation of cell division machinery. Thus, it seems appropriate to compare how cell division is realized in different microorganisms. In particular, the cell division system of Crenarchaeota lacks certain proteins found in most bacteria and Euryarchaeota, such as FtsZ, MreB or the Min system. The Sulfolobaceae family encodes functional homologs of the eukaryotic proteins vacuolar protein sorting 4 (Vps4) and endosomal sorting complex required for transport-III (ESCRT-III). ESCRT-III is essential for several eukaryotic pathways, e.g., budding of intraluminal vesicles, or cytokinesis, whereas Vps4 dissociates the ESCRT-III complex from the membrane. Cell Division A (CdvA) is required for the recruitment of crenarchaeal ESCRT-III proteins to the membrane at mid-cell. The proteins polymerize and form a smaller structure during constriction. Thus, ESCRT-III mediated cell division in Sulfolobus acidocaldarius shows functional analogies to the $Z$ ring observed in prokaryotes like Escherichia coli, which has recently begun to be reconstituted in vitro. In this short perspective, we discuss the possibility of building such an in vitro cell division system on basis of archaeal ESCRT-III.

\section{Keywords: Sulfolobus acidocaldarius, cell division, ESCRT-III, Vps4, CdvA, GUV}

\section{ARCHAEAL CELL DIVISION}

Although the basic requirements for any cell division are evident: (a) Identification of a division site, (b) targeting and assembly of elements required for membrane and cell wall constriction, and (c) coordination of constriction with genome segregation, we still lack a fundamental quantitative understanding of these processes in nearly all organisms. This can be exemplified by the open questions of how large the constricting forces actually have to be, and what brings them about. Obviously, at least the last step of membrane constriction involves membrane fusion, constituting an obvious link between division and membrane fusion machineries in general and potentially across species. Notably, recent works revealed the important roles of the archaeal homologs to the eukaryotic vacuolar protein sorting 4 (Vps4, Saci_1372, also called $\mathrm{CdvC}$ ) and endosomal sorting complex required for transport-III (ESCRT-III, Saci_1373, also called CdvB) proteins together with the protein CdvA (Saci_1374) for cell division in Sulfolobus acidocaldarius (Obita et al., 2007; Lindas etal., 2008; Samson etal., 2008, 2011; Wollert et al., 2009a,b; Wollert and Hurley, 2010) and for secretion of vesicles (Ellen et al., 2009). After the segregation of the nucleoids, a ring formed of CdvA is supposed to precede the appearance of the ESCRT-III rings, which are later disassembled by the Vps4 (Samson et al., 2011). This raises the important question whether these protein machineries may be potential candidates as constriction force generators, and for reconstituting coordinated membrane constriction with a minimal set of components, irrespective of scaling issues. In the following, we will briefly discuss the relevant modules of this machinery and their known functions.

\section{CdvA, A CYTOSKELETAL LINK BETWEEN DNA, MEMBRANE, AND ESCRT-III}

The membrane-binding cytoskeletal protein CdvA was first investigated in Metallosphaera sedula and exhibits functional homologs that are only expressed in Crenarchaeota (except Thermoproteales) and Thaumarchaeota (Moriscot et al., 2011; Samson et al., 2011). Phylogenetic analysis in NCBI databases led to a tree that is notably homogeneous within Archaea, indicating that the distribution of CdvA is no horizontal gene transfer (HGT) event (Moriscot et al., 2011), and is evolutionary lost in Thermoproteales and possibly also in Euryarchaeota (Moriscot et al., 2011).

In Sulfolobus acidocaldarius, CdvA (Saci_1374) serves as a recruitment platform for ESCRT-III (Lindas et al., 2008; Moriscot et al., 2011; Samson etal., 2011). The CdvA/ESCRT-III interface revealed a novel interaction site between the C-terminal winged Helix-like domain of crenarchaeal ESCRT-III and CdvA, which also impacts on the MIT domain of Vps4 in a similar way as in eukaryotes (Obita et al., 2007; Kieffer et al., 2008; Samson et al., 2008; Figure 1B). It was also demonstrated that the $\alpha$-helical region of CdvA supports membrane interaction, in synergy with ESCRT-III, which is responsible for the deformation of liposomes in vitro (Samson et al., 2011; Figure 1). These functions are comparable with the eukaryotic ESCRT system, where ESCRT-I and ESCRT-II facilitate scission of membrane at physiological concentrations of ESCRT-III (Wollert and Hurley, 2010).

Sulfolobus acidocaldarius produces three additional ESCRTIII-like paralogs (Saci_0451, Saci_1416, and Saci_1601) to form $40 \mathrm{~nm}$ wide membrane tubules, analogously to the eukaryotic 
A
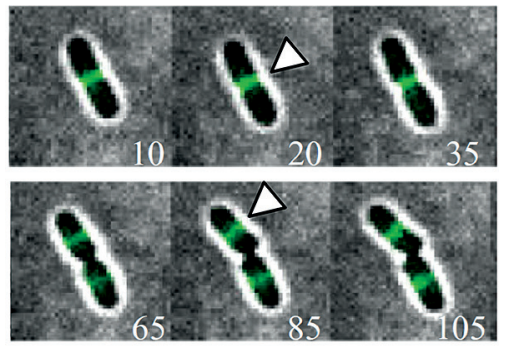

B

B MinD

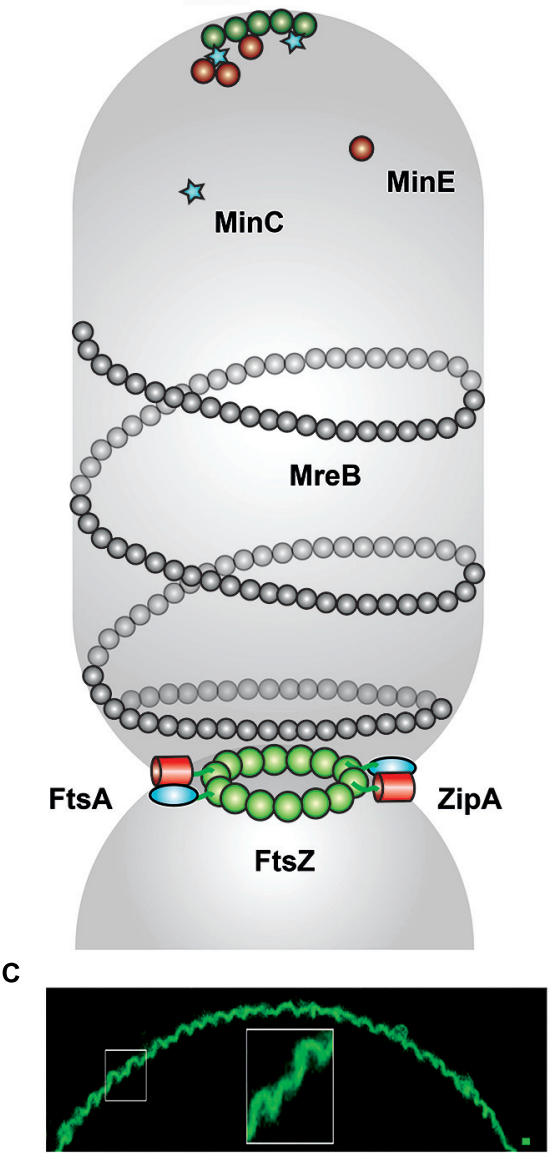

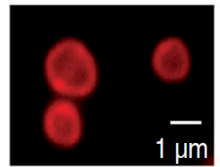
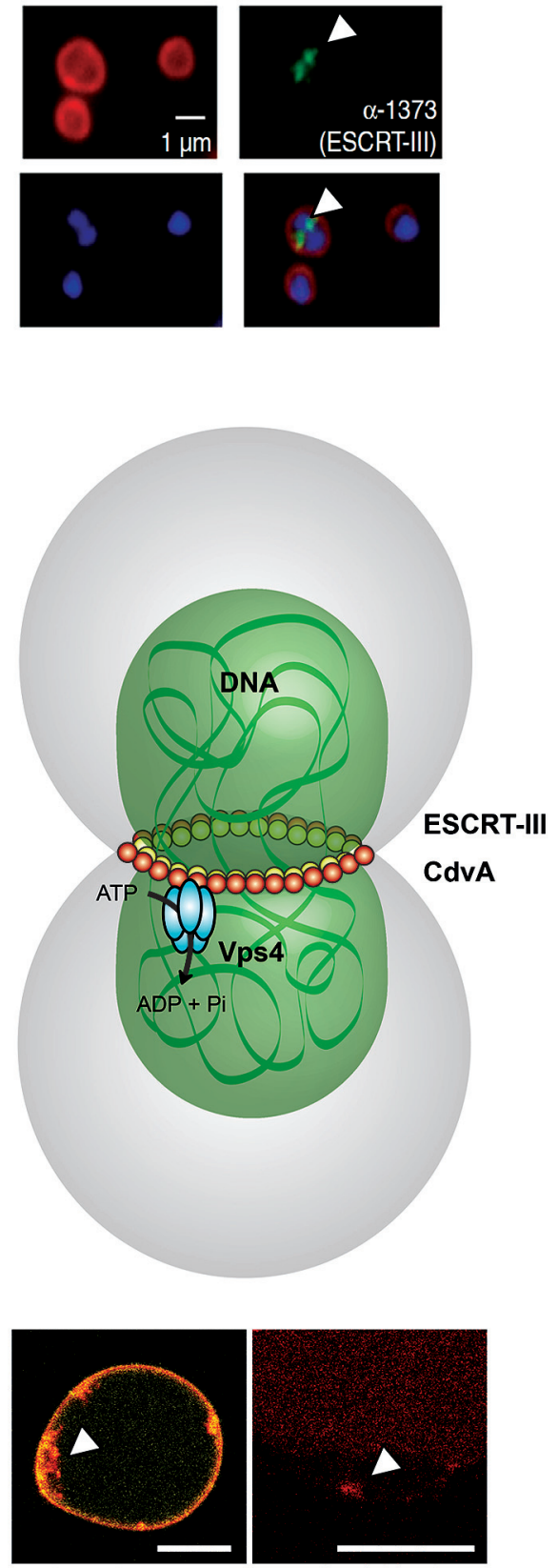

fluidity (Rico et al., 2013). MreB (gray) structures colocalize with Z ring at mid-cell (Fenton and Gerdes, 2013). Right: ESCRT-III-mediated cell division in Sulfolobus acidocaldarius. CdvA (Saci_1374, yellow) and DNA (green) build up double-helical structures horizontally to the cytokinesis region in Sulfolobus acidocaldarius (gray). ESCRT-III (Saci_1373, red) and paralogs interact with CdvA via its C-terminal winged Helix-like domain and also bind to MIT domain of the Vps4 (Saci_1372, cyan). The AAA-type ATPase Vps4 regenerates the ESCRT-III complex after cytokinesis (Moriscot et al., 2011; Samson et al., 2011). (C) Left: Membrane curvature induced in GUVs by assembly of FtsZ filaments. Under low membrane tension conditions, MTS-FtsZ-YFP showed spontaneous deformation of the GUV membranes (Arumugam et al., 2012). Right: Membrane curvature events in GUVs by the ESCRT-III machinery. By droplet emulsion transfer method CdvA, ESCRT-III (red Alexa647 labeled) and Vps4 were inserted into DOPC GUVs (yellow). The location of ESCRT-III (white arrowhead) to curved membrane suggests a function in membrane deformation event. Scale bar, $10 \mu \mathrm{m}$. 
system, after recruitment of ESCRT-III to the cell division site by CdvA (Samson and Bell, 2009; Samson et al., 2011). CdvA positions itself at the mid-cell region between segregated nucleoids, and its ring structures seem to constitute a platform for ESCRTIII rings between the nucleoids, supposedly to support their segregation. Furthermore, CdvA organizes into double-helical structures stabilized by DNA without any conventional DNAbinding motifs (Moriscot et al., 2011). The central region (residues 69-196) of CdvA in Sulfolobus acidocaldarius shows the signature of a coiled-coil motif for DNA binding and the formation of nucleoprotein filaments. The protein sequence of CdvA further suggests that the $\mathrm{N}$-terminal 70 residues might form a $\beta$-barrel with similarity to the PRC-barrel, a widespread domain implied in a number of cellular processes (Anantharaman and Aravind, 2002).

Because the double helical structure of CdvA filaments resembles actin filaments, CdvA could be a functional analog of archaeal actin. Thus, both, the morphology of CdvA filaments, and the phylogenetic analyses, support the hypothesis that it constitutes an ancient cytoskeleton protein involved in cell division. But also the alternative proposition, that FtsA is an ancient cell division protein of the actin family, which evolved into a cytoskeletal element, should be mentioned (Sanchez et al., 1994). Moreover, the formation of such helical filaments fit well with observations of CdvA structures in the cell prior to nucleoid segregation (Samson and Bell, 2009; Samson et al., 2011).

\section{ESCRT-III, A MEMBRANE SCISSION COMPLEX}

All clades of eukaryotes have ESCRTs (Dacks and Field, 2007). All Archaea having a Vps4-like ATPase and expressing ESCRT-IIIlike homologs form a closely related group, although they are lacking genes for ESCRT-0, ESCRT-I, or ESCRT-II. Moreover, one gene of an ESCRT-III-like subunit is always adjacent to the Vps4-like ATPase gene. This conserved gene cluster arrangement suggests that they encode for functional partners (Obita et al., 2007).

One major finding was that eukaryotic ESCRT-III proteins alone have the specific ability to drive the detachment of intraluminal vesicles (ILVs; Wollert et al., 2009a). For this purpose, ESCRT subunits are recruited from the cytosol to build up multivesicular bodies (MVBs), to facilitate budding of certain enveloped viruses, or to catalyze posterior steps of cytokinesis (Saksena et al., 2007; Hurley et al., 2009; Raiborg and Stenmark, 2009). ESCRTIII was identified as the minimal membrane fission machinery, which assembles a membrane neck structure during budding, and localizes between two separating daughter cells (Wollert et al., 2009a; Wollert and Hurley, 2010). Purified ESCRT-III subunits of Saccharomyces cerevisiae have been shown to facilitate budding and scission of ILVs from giant unilamellar vesicles (GUVs) at a high concentration level ( $I m$ etal., 2009; Wollert et al., 2009a).

Thus, the ESCRT-III complex obviously plays an important role in the constriction of the membrane neck structure required for complete fission (Peel et al., 2011). This ESCRT-III mediated membrane contraction resembles the membrane constriction by dynamin, but with different membrane topology. In the case of dynamin, helical structures assemble on the outside of the membrane neck to dissever endocytotic vesicles from the plasma membrane, leading to fission (Mettlen et al., 2009). In contrast, ESCRT-III seems to mount on the inner membrane neck and catalyze membrane rupture by the interplay with Vps4 ATP hydrolysis (Lata et al., 2008; Fabrikant et al., 2009; Wollert et al., 2009a; Wollert and Hurley, 2010; Peel et al., 2011). In Sulfolobus solfataricus it was shown that the C-terminal region and the residues 164-207 of ESCRT-III are essential for the direct interaction to the archaeal Vps4-like ATPase MIT domain (Obita et al., 2007).

\section{Vps4, A CELL DIVISION INVOLVED ATPase}

The eukaryotic Vps4 is the first example of an AAA-type ATPase where the oligomeric structures are important for ATP hydrolysis (Babst et al., 1998). In Saccharomyces cerevisiae, endogenous Vps4 forms stable dimers, but in the presence of ATP, these dimers oligomerize into a stable, ring-like decameric complex of five symmetric dimers (Babst et al., 1998; Obita et al., 2007). Interestingly, two features of $\mathrm{Vps} 4$ are responsible for its cycling between dimeric and oligomeric forms: the nucleotide-dependent self-affinity of the Vps4 dimers, and the regulation of ATP hydrolysis by the oligomeric state.

Nucleotide binding and hydrolysis by the AAA domain directly modifies the structure of the ATPase Vps4, and also shifts the subcellular distribution of Vps4, ESCRT-III, and Vps32/Snf7 (Babst et al., 1998). It also seems that Vps4 binds to an endosomal compartment through a coiled-coil domain, interacting with a membrane-bound protein complex of Vps24 (ESCRT-IIIlike protein) or Snf7, both also containing coiled-coil domains (Takei et al., 1995). For this purpose, Vps4 dimers in the ATPbound form are recruited from the cytosol to the endosomal membrane, which leads in turn to oligomerization of Vps4 to decamers, binding to the ESCRT-III complex (class E Vps protein complex) via coiled-coil interactions. The oligomerization of Vps4 dimers accessorily activates ATP hydrolysis, which results in a conformational change in the coiled-coil domain and in return in disassembly of the ESCRT-III complex (Babst etal., 1998).

In this context, eukaryotic Vps4 operates as an endosomalbound dissociation factor for the ESCRT-III complex by its ATPase activity (Babst et al., 1998; Peel et al., 2011). Deletion mutants and siRNA studies revealed that all human ESCRT-III and Vps4 proteins are essential for the completion of cytokinesis (Carlton and Martin-Serrano, 2007; Morita et al., 2007, 2010; Dukes et al., 2008). In Sulfolobus solfataricus, the interaction between the MIT domain of the Vps4-like protein and the C-terminal end of the ESCRT-III-like protein was demonstrated (Obita et al., 2007; Hobel et al., 2008). The isolated Vps4 MIT domain reveals only a modest affinity to ESCRT-III, but in contrast, the dodecameric Vps4 complex binds its target efficiently (Obita et al., 2007). Both Vps4-interacting ESCRT-III-related subunits, Vps2 and Did2, are required for efficient vacuolar sorting (Nickerson et al., 2006).

On the ATP hydrolysis-induced transition of Vps4 to a monomer, the low affinity of a single MIT domain for ESCRT-III would facilitate the rapid dissociation of Vps4 from ESCRTs. 
A
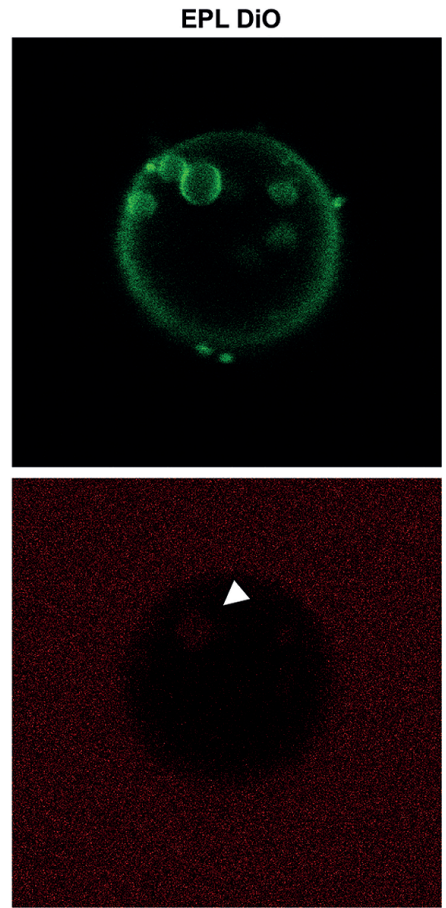

ESCRT-III Alexa647 free Cy3
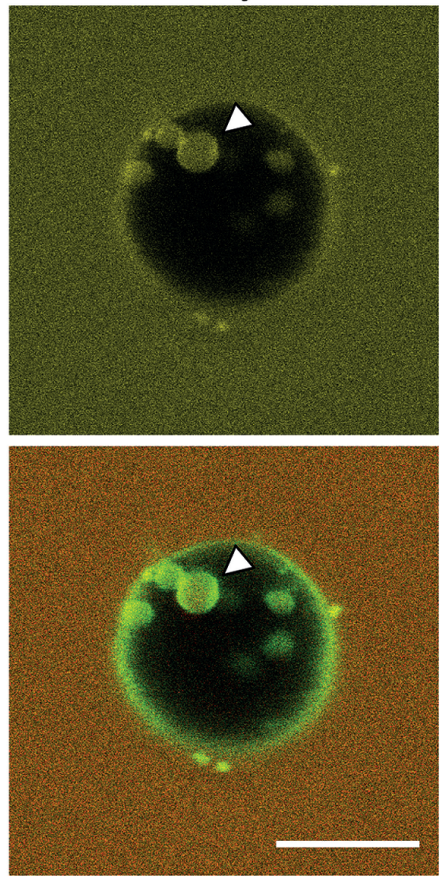

Merge

B
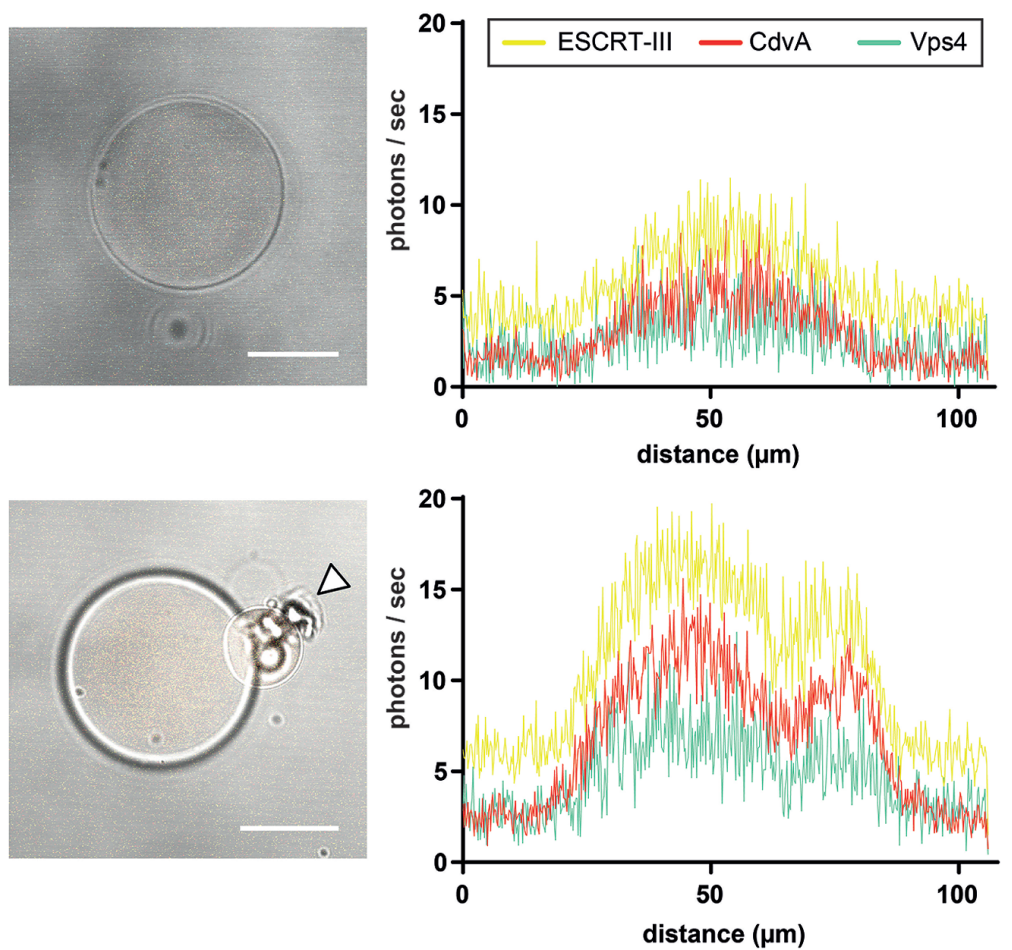

FIGURE 2 | Liposome preparation for intraluminal experiments. (A) ILV formation into EPL (Escherichia coli polar lipids) GUVs in presence of ESCRT-III and Vps4. The GUV membrane is stained green with DiO. Red labeled ESCRT-III proteins (Alexa647), unstained Vps4 proteins and free Cy3 were added to the extraluminal buffer of the EPL GUVs. After ILV formation of the GUV membrane ESCRT-III as well as the soluble marker Cy3 could be visualized in the vesicles (white arrowheads). Scale bar, $10 \mu \mathrm{m}$. (B) De novo protein production in GUVs via protein synthesis kit within $3 \mathrm{~h}$. Left figures reveal brightfield images of a GUV with recombinant CdvA-mCherry, Vps4-AmCyan1 and ESCRT-III-zYellow1 plasmids in combination with a protein synthesis kit included. The low fluorescence level (histogram in upper right) of all three proteins indicates the beginning of de novo production. After $3 \mathrm{~h}$ of incubation the fluorescence of all proteins are increased (histogram in lower right) and it could be observed a budding event at GUV (lower left image, white arrowhead). Scale bar, $10 \mu \mathrm{m}$. 
The ESCRT-III complex triggers its own disassembly. It recruits monomeric or dimeric Vps4 to membranes, on which the multimeric ESCRT-III aggregate facilitates the assembly of the active Vps4 oligomer. Once the Vps4 rings have assembled, ATP hydrolysis would lead to the disassembly of both the ATPase rings and the ESCRT-III complex (Obita et al., 2007).

\section{RECONSTITUTION PERSPECTIVES}

With respect to a possible reconstitution of the division process from the bottom-up, bacteria and Archaea promise more restricted and practically manageable modules, compared to the vastly complex cytokinetic machineries in eukaryotes. In recent years, our group has explored the reconstitution of the Escherichia coli cell division system, module by module. We successfully reproduced in vitro the emergence of self-organized waves, and lately, oscillations, of the Min protein machinery positioning the contractile $\mathrm{Z}$ ring (Loose et al., 2008; Zieske and Schwille, 2013). The MinCDE system does likely not take part in the constriction, but is an essential septum positioning device. E. coli min deletion mutants are still viable, but with fluctuating localization of cytokinesis (Teather et al., 1974; de Boer et al., 1989, 1990, 1992). We furthermore investigated the assembly of FtsZ proto-rings on membranes and their curvature sensing and inducing propensity (Arumugam et al., 2012), however with no indication so far that the contractile force required for cell division could result from FtsZ only. Several other research groups presently attempt the reconstitution and assembly of bacterial divisome, based mainly on FtsZ and its anchors, in vesicles (Osawa et al., 2008; Jimenez et al., 2011; Cabre et al., 2013; Osawa and Erickson, 2013). However, considering that a progressively contractile role of FtsZ could so far not be established, in spite of its membrane-sculpting ability, it seems appropriate to explore alternative membranetransforming machineries to accomplish compartment division. Thus, we presently investigate in a cell-free system the interaction of the three archaeal cell division proteins discussed above: Vps4, ESCRT-III, and CdvA of Sulfolobus acidocaldarius. As a first step, we accomplished their intra- and extra luminal reconstitution into artificial constructed liposomes, in a bottom-up approach.

Confocal imaging reveals extraluminal membrane binding and ILV formation into EPL (Escherchia coli polar lipids) GUVs in presence of ESCRT-III and Vps4 (Figure 2A). Intraluminal experiments with the ESCRT-III machinery were designed following the so-called droplet method, in which water-in-oil droplets are converted into liposomes through sonication and two-step centrifugation (Pontani et al., 2009; van Swaay and deMello, 2013). This method is compatible with the use of purified or de novo synthesized proteins within the liposomes. Thus, we may study the function and interplay of Vps4, ESCRT-III, and CdvA, either using purified proteins, or by intraluminal de novo protein synthesis. In both cases, the protein abundance can be visualized by confocal imaging via LSM780 microscope (Zeiss). Therefore, the genes of Vps4, ESCRT-III, and CdvA (saci_1372 to saci_1374) were ligated into recombinant fluorescence plasmids. Purified cell division proteins were labeled either C-terminally or N-terminally with one fluorophor (mCherry, Yellow1, or AmCyan1; Figure 2B). GUVs are useful model systems to reveal membrane transformation by the expressed proteins. Their mean diameter $(1-50 \mu \mathrm{m})$ can be made comparable to the average size of Sulfolobus acidocaldarius cells $(1-2 \mu \mathrm{m})$. Alternatively, $80-800 \mathrm{~nm}$ sized large unilamellar vesicles (LUVs) may be explored in prospective studies, which are, however, much less amenable to standard light microscopy observation.

Remarkably, specific deformation of GUVs after coreconstitution of all three proteins could be observed reproducibly. This phenomenon will now be subject to a more quantitative analysis, addressing the range of shapes and structures formed on and from the membranes. We will address the influence of electric charge polarity and lipid composition of the membrane vesicles on curvature and general abundance of budding events. Further, the interactions of crenarchaeal ESCRT-III-like paralogs (Saci_0451, Saci_1416, and Saci_1601) should be investigated, to address the question whether or not these paralogs are essential, or only required for fine-tuning of cell division in Sulfolobus acidocaldarius. To access spatial scales below the optical resolution limit, we have begun to visualize the interaction of DNA with the CdvA complex, leading to its double helical structure, by super-resolution microscopy [stochastic optical reconstruction microscopy (STORM)] and atomic force microscopy (AFM).

Clearly, these data constitute only the beginning of a series of experiments, exploring the potential of ESCRT-III mediated (proto-) cell division in a minimal system. Besides the fundamental mechanistic aspects of membrane transformation that may be addressed by such a bottom-up reconstitution, also the phylogenetic and evolutionary history of the division machinery in Crenarchaeota may be explored. This may answer the questions on diversity of the FtsZ-based systems, and why ESCRT-III mediated cell division is now mainly found in thermophilic and acidothermophilic Crenarchaeota and Thaumarchaeota. The linkage between cell division in Archaea and ESCRT-III dependent biogenesis of MVBs in eukaryotes is a fascinating evolutionary aspect (Poole, 2006; Hanson et al., 2008). Taken together, we will explore the scaling variability of ESCRT-III mediated constriction, and potentially, facilitate ESCRT-III based division of larger membrane compartments, to reveal its potential suitability as a truly "archetypical" cell division machinery to be assembled from the bottom-up.

\section{REFERENCES}

Anantharaman, V., and Aravind, L. (2002). The PRC-barrel: a widespread, conserved domain shared by photosynthetic reaction center subunits and proteins of RNA metabolism. Genome Biol. 3, RESEARCH0061. doi: 10.1186/gb-2002-3-11research0061

Arumugam, S., Chwastek, G., Fischer-Friedrich, E., Ehrig, C., Mönch, I., and Schwille, P. (2012). Surface topology engineering of membranes for the mechanical investigation of the tubulin homologue FtsZ. Angew. Chem. Int. Ed. Engl. 51, 11858-11862. doi: 10.1002/anie.201204332

Babst, M., Wendland, B., Estepa, E. J., and Emr, S. D. (1998). The Vps4p AAA ATPase regulates membrane association of a $\mathrm{Vps}$ protein complex required for normal endosome function. EMBO J. 17, 2982-2993. doi: 10.1093/emboj/17.11.2982

Buss, J., Coltharp, C., Huang, T., Pohlmeyer, C., Wang, S. C., Hatem, C., et al. (2013). In vivo organization of the FtsZ-ring by ZapA and ZapB revealed by quantitative super-resolution microscopy. Mol. Microbiol. 89, 1099-1120. doi: $10.1111 / \mathrm{mmi} .12331$

Cabre, E. J., Sánchez-Gorostiaga, A., Carrara, P., Ropero, N., Casanova, M., Palacios, P., et al. (2013). Bacterial division proteins FtsZ and ZipA induce vesicle 
shrinkage and cell membrane invagination. J. Biol. Chem. 288, 26625-26634. doi: 10.1074/jbc.M113.491688

Carlton, J. G., and Martin-Serrano, J. (2007). Parallels between cytokinesis and retroviral budding: a role for the ESCRT machinery. Science 316, 1908-1912. doi: $10.1126 /$ science. 1143422

Dacks, J. B., and Field, M. C. (2007). Evolution of the eukaryotic membranetrafficking system: origin, tempo and mode. J. Cell Sci. 120, 2977-2985. doi: $10.1242 /$ jcs. 013250

de Boer, P. A., Crossley, R. E., and Rothfield, L. I. (1989). A division inhibitor and a topological specificity factor coded for by the minicell locus determine proper placement of the division septum in E. coli. Cell 56, 641-649. doi: 10.1016/00928674(89)90586-2

de Boer, P. A., Crossley, R. E., and Rothfield, L. I. (1990). Central role for the Escherichia coli minC gene product in two different cell division-inhibition systems. Proc. Natl. Acad. Sci. U.S.A. 87, 1129-1133. doi: 10.1073/pnas.87. 3.1129

de Boer, P. A., Crossley, R. E., and Rothfield, L. I. (1992). Roles of MinC and MinD in the site-specific septation block mediated by the MinCDE system of Escherichia coli. J. Bacteriol. 174, 63-70.

Dukes, J. D., Richardson, J. D., Simmons, R., and Whitley, P. (2008). A dominantnegative ESCRT-III protein perturbs cytokinesis and trafficking to lysosomes. Biochem. J. 411, 233-239. doi: 10.1042/BJ20071296

Ellen, A. F., Albers, S. V., Huibers, W., Pitcher, A., Hobel, C. F., Schwarz, H., etal. (2009). Proteomic analysis of secreted membrane vesicles of archaeal Sulfolobus species reveals the presence of endosome sorting complex components. Extremophiles 13, 67-79. doi: 10.1007/s00792-0080199-x

Fabrikant, G., Lata, S., Riches, J. D., Briggs, J. A., Weissenhorn, W., and Kozlov, M. M. (2009). Computational model of membrane fission catalyzed by ESCRT-III. PLoS Comput. Biol. 5:e1000575. doi: 10.1371/journal.pcbi.10 00575

Fenton, A. K., and Gerdes, K. (2013). Direct interaction of FtsZ and MreB is required for septum synthesis and cell division in Escherichia coli. EMBO J. 32, 1953-1965. doi: 10.1038/emboj.2013.129

Hanson, P. I., Roth, R., Lin, Y., and Heuser, J. E. (2008). Plasma membrane deformation by circular arrays of ESCRT-III protein filaments. J. Cell Biol. 180, 389-402. doi: $10.1083 /$ jcb.200707031

Hobel, C. F., Albers, S. V., Driessen, A. J., and Lupas, A. N. (2008). The Sulfolobus solfataricus AAA protein Sso0909, a homologue of the eukaryotic ESCRT Vps4 ATPase. Biochem. Soc. Trans. 36, 94-98. doi: 10.1042/ BST0360094

Hurley, J. H., Im, Y. J., Lee, H. H., Ren, X., Wollert, T., and Yang, D. (2009). Piecing together the ESCRTs. Biochem. Soc. Trans. 37, 161-166. doi: 10.1042/BST0370161

Im, Y. J., Wollert, T., Boura, E., and Hurley, J. H. (2009). Structure and function of the ESCRT-II-III interface in multivesicular body biogenesis. Dev. Cell 17, 234-243. doi: 10.1016/j.devcel.2009.07.008

Jimenez, M., Martos, A., Vicente, M., and Rivas, G. (2011). Reconstitution and organization of Escherichia coli proto-ring elements (FtsZ and FtsA) inside giant unilamellar vesicles obtained from bacterial inner membranes. J. Biol. Chem. 286, 11236-11241. doi: 10.1074/jbc.M110.194365

Kieffer, C., Skalicky, J. J., Morita, E., De Domenico, I., Ward, D. M., Kaplan, J., et al. (2008). Two distinct modes of ESCRT-III recognition are required for VPS4 functions in lysosomal protein targeting and HIV-1 budding. Dev. Cell 15, 62-73. doi: 10.1016/j.devcel.2008.05.014

Lata, S., Schoehn, G., Jain, A., Pires, R., Piehler, J., Gottlinger, H. G., et al. (2008). Helical structures of ESCRT-III are disassembled by VPS4. Science 321, 13541357. doi: 10.1126/science. 1161070

Lindas, A. C., Karlsson, E. A., Lindgren, M. T., Ettema, T. J., and Bernander, R. (2008). A unique cell division machinery in the Archaea. Proc. Natl. Acad. Sci. U.S.A. 105, 18942-18946. doi: 10.1073/pnas.0809 467105

Loose, M., Fischer-Friedrich, E., Ries, J., Kruse, K., and Schwille, P. (2008). Spatial regulators for bacterial cell division self-organize into surface waves in vitro. Science 320, 789-792. doi: 10.1126/science. 1154413

Mettlen, M., Pucadyil, T., Ramachandran, R., and Schmid, S. L. (2009). Dissecting dynamin's role in clathrin-mediated endocytosis. Biochem. Soc. Trans. 37, 10221026. doi: 10.1042/BST0371022
Moriscot, C., Gribaldo, S., Jault, J. M., Krupovic, M., Arnaud, J., Jamin, M., et al. (2011). Crenarchaeal CdvA forms double-helical filaments containing DNA and interacts with ESCRT-III-like CdvB. PLoS ONE 6:e21921. doi: 10.1371/journal.pone.0021921

Morita, E., Colf, L. A., Karren, M. A., Sandrin, V., Rodesch, C. K., and Sundquist, W. I. (2010). Human ESCRT-III and VPS4 proteins are required for centrosome and spindle maintenance. Proc. Natl. Acad. Sci. U.S.A. 107, 12889-12894. doi: 10.1073/pnas. 1005938107

Morita, E., Sandrin, V., Chung, H. Y., Morham, S. G., Gygi, S. P., Rodesch, C. K., et al. (2007). Human ESCRT and ALIX proteins interact with proteins of the midbody and function in cytokinesis. EMBO J. 26, 4215-4227. doi: 10.1038/sj.emboj.7601850

Nickerson, D. P., West, M., and Odorizzi, G. (2006). Did2 coordinates Vps4mediated dissociation of ESCRT-III from endosomes. J. Cell Biol. 175, 715-720. doi: $10.1083 /$ jcb. 200606113

Obita, T., Saksena, S., Ghazi-Tabatabai, S., Gill, D. J., Perisic, O., Emr, S. D., et al. (2007). Structural basis for selective recognition of ESCRT-III by the AAA ATPase Vps4. Nature 449, 735-739. doi: 10.1038/nature06171

Osawa, M., and Erickson, H. P. (2013). Liposome division by a simple bacterial division machinery. Proc. Natl. Acad. Sci. U.S.A. 110, 11000-11004. doi: 10.1073/pnas. 1222254110

Osawa, M., Anderson, D. E., and Erickson, H. P. (2008). Reconstitution of contractile FtsZ rings in liposomes. Science 320, 792-794. doi: 10.1126/science.11 54520

Peel, S., Macheboeuf, P., Martinelli, N., and Weissenhorn, W. (2011). Divergent pathways lead to ESCRT-III-catalyzed membrane fission. Trends Biochem. Sci. 36, 199-210. doi: 10.1016/j.tibs.2010.09.004

Pontani, L. L., van der Gucht, J., Salbreux, G., Heuvingh, J., Joanny, J. F., and Sykes, C. (2009). Reconstitution of an actin cortex inside a liposome. Biophys. J. 96, 192-198. doi: 10.1016/j.bpj.2008.09.029

Poole, A. M. (2006). Did group II intron proliferation in an endosymbiontbearing archaeon create eukaryotes? Biol. Direct 1, 36. doi: 10.1186/1745-61 50-1-36

Raiborg, C., and Stenmark, H. (2009). The ESCRT machinery in endosomal sorting of ubiquitylated membrane proteins. Nature 458, 445-452. doi: 10.1038/nature07961

Rico, A. I., Krupka, M., and Vicente, M. (2013). In the beginning, Escherichia coli assembled the proto-ring: an initial phase of division. J. Biol. Chem. 288, 20830-20836. doi: 10.1074/jbc.R113.479519

Saksena, S., Sun, J., Chu, T., and Emr, S. D. (2007). Emr, ESCRTing proteins in the endocytic pathway. Trends Biochem. Sci. 32, 561-573. doi: 10.1016/j.tibs.2007.09.010

Samson, R. Y., and Bell, S. D. (2009). Ancient ESCRTs and the evolution of binary fission. Trends Microbiol. 17, 507. doi: 10.1016/j.tim.2009. 08.003

Samson, R. Y., Obita, T., Freund, S. M., Williams, R. L., and Bell, S. D. (2008). A role for the ESCRT system in cell division in archaea. Science 322, 1710-1713. doi: $10.1126 /$ science. 1165322

Samson, R. Y., Obita, T., Hodgson, B., Shaw, M. K., Chong, P. L., Williams, R. L., et al. (2011). Molecular and structural basis of ESCRT-III recruitment to membranes during archaeal cell division. Mol. Cell 41, 186-196. doi: 10.1016/j.molcel.2010.12.018

Sanchez, M., Valencia, A., Ferrandiz, M. J., Sander, C., and Vicente, M. (1994). Correlation between the structure and biochemical activities of FtsA, an essential cell division protein of the actin family. EMBO J. 13, 4919-4925.

Takei, K., McPherson, P. S., Schmid, S. L., and De Camilli, P. (1995). Tubular membrane invaginations coated by dynamin rings are induced by GTP-gamma $S$ in nerve terminals. Nature 374, 186-190. doi: 10.1038/374186a0

Teather, R. M., Collins, J. F., and Donachie, W. D. (1974). Quantal behavior of a diffusible factor which initiates septum formation at potential division sites in Escherichia coli. J. Bacteriol. 118, 407-413.

van Swaay, D., and deMello, A. (2013). Microfluidic methods for forming liposomes. Lab Chip 13, 752-767. doi: 10.1039/c2lc41121k

Wollert, T., and Hurley, J. H. (2010). Molecular mechanism of multivesicular body biogenesis by ESCRT complexes. Nature 464, 864-869. doi: 10.1038/nature08849

Wollert, T., Wunder, C., Lippincott-Schwartz, J., and Hurley, J. H. (2009a). Membrane scission by the ESCRT-III complex. Nature 458, 172-177. doi: $10.1038 /$ nature 07836 
Wollert, T., Yang, D., Ren, X., Lee, H. H., Im, Y. J., and Hurley, J. H. (2009b). The ESCRT machinery at a glance. J. Cell Sci. 122, 2163-2166. doi: 10.1242/jcs.029884 Zieske, K., and Schwille, P. (2013). Reconstitution of pole-to-pole oscillations of min proteins in microengineered polydimethylsiloxane compartments. Angew. Chem. 52, 459-462. doi: 10.1002/anie.201207078

Conflict of Interest Statement: The authors declare that the research was conducted in the absence of any commercial or financial relationships that could be construed as a potential conflict of interest.

Received: 04 December 2013; accepted: 11 May 2014; published online: 04 June 2014.
Citation: Härtel T and Schwille P (2014) ESCRT-III mediated cell division in Sulfolobus acidocaldarius - a reconstitution perspective. Front. Microbiol. 5:257. doi 10.3389/fmicb.2014.00257

This article was submitted to Microbial Physiology and Metabolism, a section of the journal Frontiers in Microbiology.

Copyright (C) 2014 Härtel and Schwille. This is an open-access article distributed under the terms of the Creative Commons Attribution License (CC BY). The use, distribution or reproduction in other forums is permitted, provided the original author(s) or licensor are credited and that the original publication in this journal is cited, in accordance with accepted academic practice. No use, distribution or reproduction is permitted which does not comply with these terms. 